\title{
Measurement of residual stresses around the notch of tensile specimens of the high-Cr tempered martensitic steel F82H-mod
}

\author{
Nazar Ilchuk ${ }^{1, a^{*}}$, Philippe Spätig ${ }^{2, b}$, Vadim Davydov ${ }^{3, c}$ \\ and Steven Van Petegem ${ }^{3, d}$ \\ ${ }^{1}$ Fusion Technology-Materials, CRPP EPFL, Association EURATOM-Confédération Suisse, 5232 \\ Villigen PSI, Switzerland \\ ${ }^{2}$ Laboratory for Nuclear Materials, Nuclear Energy and Safety Research Department, 5232 Villigen \\ PSI, Switzerland \\ ${ }^{3}$ Materials Science and Simulations, NUM ASQ, 5232 Villigen PSI, Switzerland \\ anazar.ilchuk@psi.ch, ${ }^{b}$ philippe.spatig@psi.ch, ${ }^{\mathrm{C}}$ vadim.davydov@psi.ch, \\ dsteven.vanpetegem@psi.ch
}

Keywords: tempered martensitic steel, notched tensile specimen, residual elastic strains and stresses, neutron diffraction.

\begin{abstract}
Residual elastic strains were measured by neutron diffraction using POLDI materials science diffractometer at PSI-Switzerland on pre-loaded notched flat tensile specimens made of the high-chromium tempered martensitic steel F82H-mod steel. To calculate the residual stresses using Hooke's equation, three perpendicular components of the residual strain field were determined. The measured residual strains and stresses were compared with those deduced from finite element simulation calculations. A very good agreement was found for the strains in the loading plane of the specimen while a somewhat larger discrepancy was observed for the out-of-plane residual strain, which was tentatively attributed to an uncertainty in the initial lattice spacing in that direction.
\end{abstract}

\section{Introduction}

The high-chromium reduced-activation tempered martensitic steels are among the most advanced structural materials for the future fusion reactors [1]. These steels have a body centered cubic $(b c c)$ structure and share with other $b c c$ alloys several similar characteristics. In particular, they exhibit a ductile-to-brittle fracture mode transition from high-temperature micro-void coalescence to low-temperature quasi-cleavage [2]. It is well known that the load bearing capacity of a component with a stress concentrator can be strongly affected (increased or decreased) if a residual stress field is introduced near the stress concentrator: this technique is called warm prestressing WPS [3]. While a great deal of studies on WPS have been done on reactor pressure vessel steel in the brittle regime, there is practically no investigations on WPS published as far as the reduced activation tempered martensitic steels are concerned. Recently, we have initiated some work to investigate the WPS effect on pre-cracked subsized compact tension specimens of the tempered martensitic steel Eurofer97 developed for fusion reactor applications [4]. Other research activities have also been undertaken to quantify the effect of the residual compressive stresses on notch toughness introduced by pre-loading at room temperature notched flat tensile specimens. In this paper, we report preliminary results obtained with the neutron diffraction POLDI facility at PSI, used to measured residual elastic strain in the tempered martensitic steel F82H-mod.

\section{Material, Specimen Geometry and Tensile Test}

The material studied is the $\mathrm{F} 82 \mathrm{H}-$ mod steel that contains $7.65 \mathrm{wt} \% \mathrm{Cr}, 2 \mathrm{wt} \% \mathrm{~W}, 0.1 \mathrm{wt} \% \mathrm{C}$ and a total of less than $1 \mathrm{wt} \% \mathrm{Mn}, \mathrm{Mo}, \mathrm{V}, \mathrm{Ta}, \mathrm{Ti}, \mathrm{Si}$, with the balance Fe. The steel was produced by NKK Corporation under the sponsorship of Japan Atomic Energy Research Institute and was heattreated by normalizing at $1313 \mathrm{~K}$ for $0.5 \mathrm{~h}$ and tempering at $1013 \mathrm{~K}$ for $2 \mathrm{~h}$ [5]. 
In order to introduce a residual elastic strain field around a notch, notched flat tensile specimens were pre-loaded in tension at room temperature in the macro-plastic regime and then unloaded. A picture of the specimens used in this study is shown in Fig. 1 a). The specimen thickness (notch front length) is $7 \mathrm{~mm}$, the total width is $10 \mathrm{~mm}$, the ligament length (between the two facing notch roots) is $5 \mathrm{~mm}$ and the notch radii were $0.5 \mathrm{~mm}$. On the one hand, the specimen geometry was specially designed to avoid a too large residual elastic strain gradient in the vicinity of the notch, which could not be measured with neutron stress diffractometers with highest spatial resolution. On the other hand, the pre-load level was chosen to introduce a sufficiently high residual elastic strain field to be measured. The final design of the specimen and the pre-load level were actually determined on the basis of preliminary finite element simulations. The specimen was pre-loaded with an electro-mechanical testing machine Schenk RMC100. A clip gage with an initial opening of $10 \mathrm{~mm}$ was attached to the specimen, with the two knives of the clip gage being symmetrically positioned with respect to the notch plane. The displacement reported below for the loaddisplacement curve corresponds to the opening of the clip gage.

\section{Residual Stress Measurement with POLDI Diffractometer}

The residual elastic strains were measured using neutron diffraction (ND) technique at Time-ofFlight (ToF) POLDI diffractometer located at Paul Scherrer Institute [6]. The principle of residual strain determination using that diffraction method is based upon the measurement of interplanar distances between various crystallographic planes. Owing to this intrinsic physical property of materials to diffract neutrons, and to the high penetration of neutrons into materials (up to $\sim 3 \mathrm{~cm}$ in steels), this method allows the detection of residual elastic strains deep beneath the surface. The nominal sampling volume inside of the specimen was defined by cadmium diaphragms and collimators. The output of the measurements performed by this method is a component of elastic strain that is a relative value and which is specific for every crystallographic plane due to the elastic anisotropy in a crystal. In materials with $b c c$ structure only three orthogonal principal strain components have to be measured. For the current case, due to the small employed gauge volume and to the relatively large neutron path length through the examined material, only $\{211\}$ family of crystallographic lattice planes were collected with sufficient intensity, and thus could be reliably used for stress determination. The residual elastic strains around the notch after the pre-deformation were measured on one specimen. To catch the strain gradient in the vicinity of the notch, a relatively small diffraction volume was selected for the measurement: $0.6 \times 0.6 \times 2.5 \mathrm{~mm}^{3}$ for the radial (x) and axial (y) direction while a volume of $0.6 \times 0.6 \times 0.6 \mathrm{~mm}^{3}$ was considered for the outof-plane direction (z) (see Fig. 1 b)). Three components of residual elastic strain, $\varepsilon_{x x}, \varepsilon_{y y}$, $\varepsilon_{z z}$, were measured along the centerline starting from the specimen center towards the root of the notch as indicated in Fig. 1 b) by the red crosses. The spacing between the data points along the scan is 0.4 $\mathrm{mm}$. Finally, to convert lattice strain values into stress, the generalized form of Hooke's equation has to be used, which has for $\sigma_{\mathrm{xx}}$ component the form:

$$
\sigma_{x x}=\frac{E_{h k l}}{\left(1+v_{h k l}\right)}\left[\varepsilon_{x x}+\frac{v_{h k l}}{\left(1-2 v_{h k l}\right)}\left(\varepsilon_{x x}+\varepsilon_{y y}+\varepsilon_{z z}\right)\right]
$$

Where $\varepsilon_{x x}, \varepsilon_{y y}, \varepsilon_{z z}$ are the normal strain components obtained from a particular crystal lattice plane $\{\mathrm{hkl}\}$. By means of permutation of the indices, the remainder $\sigma_{y y}$ and $\sigma_{z z}$ stress components can be obtained. $E_{h k l}$ and $v_{h k l}$ here are the diffraction plane-specific Young's modulus and Poisson's ratio respectively, or also known as diffraction elastic constants [7]. Using the tabled values of the elastic constants for $\{211\}$ lattice plane in $b c c \alpha-F e$ material [7], the Kröner model was considered as the most appropriate that takes a proper account for the interaction between a grain and its surroundings. According to this model, the following elastic constants $E_{211}=225.5 \mathrm{GPa}$ and $v_{211}=$ 0.28 were employed in stress determination. 


\section{Finite Element Simulations}

3D finite element (FE) model of notched tensile specimen was developed using ABAQUS software application. F82H-mod steel was modeled as an isotropic linear elastic-plastic material with elastic properties described by Young's modulus $(E=210 \mathrm{GPa})$ and Poisson's ratio $(v=0.3)$. The rate-independent plasticity was defined by the associated plastic flow $\sigma\left(\varepsilon_{p l}\right)$ given as a tabular function of plastic strain. The model of notched tensile specimen used was simulated as one eighth of the real geometry taking advantage of its symmetry as illustrated in Fig. 1 a), which shows the specimen-model geometry. The model was meshed with 26536 elements of the type a 4-node linear tetrahedron and 50790 elements of the type 8-node linear brick, reduced integration.

The calculated elastic strain values reported hereafter are the volume-averaged values over the same volume as the gauge volume of the neutron diffraction experimental setup.

a)

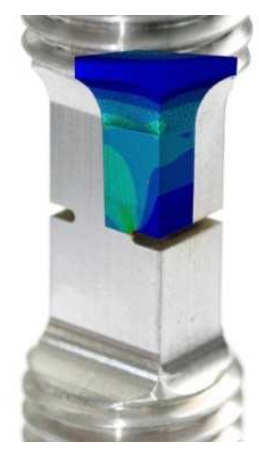

b)

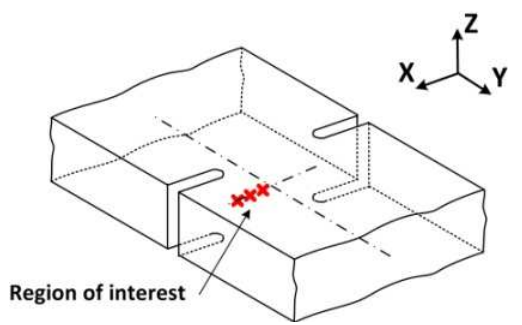

c)

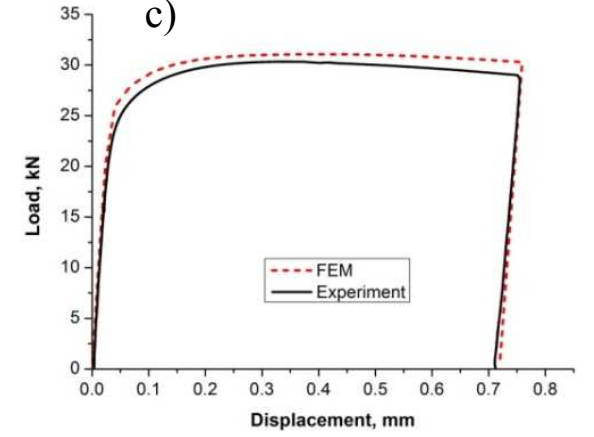

Fig. 1: a) Notched tensile specimen with FEM model, b) red crosses indicating the positions of the measurement from the specimen center, c) experimental and FEM calculated curves.

\section{Experimental Results}

The experimental load-displacement curve of notched tensile specimen tested at room temperature is presented in Fig. $1 \mathrm{c}$ ) along with the calculated one by finite element simulations. While the experimental curve is a little lower than the simulated one, a relatively good match was obtained. Owing to the stress/strain gradient that develops during the deformation around the notch, residual stresses/strains build up upon unloading of the specimen in the notch region. As a matter of fact, a triaxial stress state is created. Therefore, three components of residual elastic strain fields $\left(\varepsilon_{x x}, \varepsilon_{y y}, \varepsilon_{z z}\right)$ have to be measured to determine the residual stress components. $\varepsilon_{x x}, \varepsilon_{y y}, \varepsilon_{z z}$ were measured along the centerline starting from the specimen center towards the root of the notch as indicated in Fig. 1 b) by the red crosses. The measured three components of the residual elastic strains are plotted in Fig. 2 a), b) c) where they are compared with those obtained from the FE simulations. One can see that the measured $\varepsilon_{x x}, \varepsilon_{y y}$ are very close to the calculated ones, being practically all within the experimental determination uncertainty. However, the correspondence between the measured and calculated data of $\varepsilon_{z z}$ is rather poor. That discrepancy may be caused by the experimental procedure used here where the $d_{0}$ value (interplanar spacing) of the stress-free sample was measured only in the y-direction. It was then assumed that the material is fully isotropic and consequently that $d_{0}$ value is the same for $x$ - and $z$-directions. However, it is quite plausible that during the initial rolling of the F82H-mod plates some additional residual stresses have been introduced. Therefore, the reference $d_{0}$ value should be measured in each direction for which the lattice strains are investigated. Due to space limitation, we show only the residual axial stress in Fig. 2 d). The pre-loading condition selected has introduced a large nominal tensile residual stress of about $400 \mathrm{MPa}$ perpendicular to the notch plane that is expected to modify significantly the final fracture load at lower testing temperature. We emphasize that the residual stresses were measured along the middle centerline. Due to the large plastic deformation around the notch (no plane-strain), the residual stresses are through-thickness $(x-z$ plane in Fig. $1 \mathrm{~b})$ ) dependent. This point is illustrated in Fig. 2 d), where we add the free surface $\sigma_{y y}$ profile (data not averaged over the gauge volume), 
showing that tensile dominant stresses in the middle are balanced by compressive stresses in the outer part of the specimen.
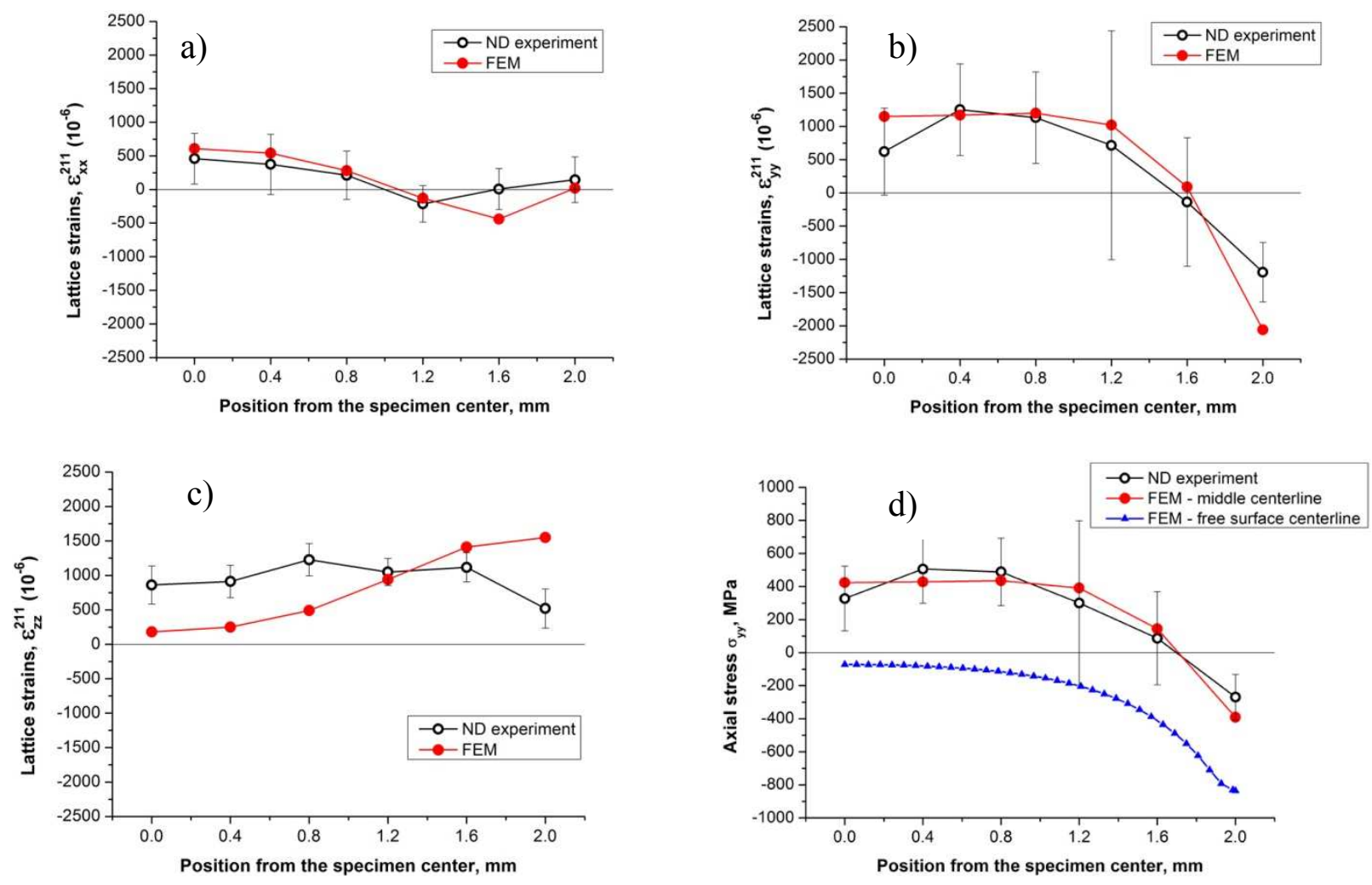

Fig. 2: a), b) and c) $\varepsilon_{x x}, \varepsilon_{y y}, \varepsilon_{z z}$ residual elastic strains and d) $\sigma_{y y}$ axial residual stress around notch.

\section{Summary}

This paper reports preliminary results of neutron diffraction measurement performed to determine residual strain/stress fields ahead of the notch of pre-loaded flat tensile specimens made of the reduced activation F82H-mod steel. This measurement was undertaken within the frame of a study to assess the effects of warm-pressing on the notch and crack toughness of tempered martensitic steels. Finite element simulations were run to supplement the experimental data. Among the three perpendicular strain components measured, two were found to be very close to the calculated ones by finite element simulation, while one exhibits more discrepancy that may be associated with a pre-existing residual stress in the as-received F82H-mod steel.

\section{References}

[1] H. Tanigawa et al., J. Nuc. Mat. 417 (2011) 9-15

[2] G. Odette, J. Nuc. Mat. 212-215 (1994) 45-51

[3] D. J. Smith and S. J. Garwood, Int. J. Pres. Ves. \& Piping, 41 (1990) 255-296

[4] N. Ilchuk, L. Commin, P. Spätig and G. R. Odette, accepted for publication in Fusion Eng. Des.

[5] M. Tamura, H. Hayakawa, M. Tanimura, A. Hishinuma, T. Kondo, J. Nuc. Mat. 141-143 (1986) 1067-1073

[6] U. Stuhr, H. Spitzer, J. Egger, A. Hofer, P. Rasmussen, D. Graf, A. Bollhalder, M. Schild, G. Bauer, W. Wagner: Nuclear Instruments and Methods in Physics Research A, 545 (2005) 330338

[7] M.T. Hutchings, P.J. Withers, T.M. Holden, T. Lorentzen, Introduction to the characterization of the residual stress by neutron diffraction, Taylor \& Francis Group, Boca Raton, FL, 2005. 